\title{
Does Lower Mantle Tomography Indicate Temperature Variations That Affect the Core?
}

\author{
Frank STACEY \\ Department of Physics, The University of Queensland, Brisbane 4072, Australia
}

(Received October 31, 1992; Revised March 12, 1993; Accepted March 30, 1993)

\begin{abstract}
A new assessment of the thermodynamic properties of the lower mantle yields a more secure value than hitherto for the adiabatic Anderson-Grüneisen parameter at a nominal depth of $1771 \mathrm{~km}$,

$$
\delta_{S}=\left(\frac{\partial \ln K_{S}}{\partial \ln \rho}\right)=\frac{1}{\alpha_{V} K_{S}}\left(\frac{\partial K_{S}}{\partial T}\right)_{P}=2.4 .
$$

The corresponding temperature dependence of rigidity is not well constrained but for the purpose of calculations is assumed to be

$$
\epsilon=\frac{1}{\alpha_{V} \mu}\left(\frac{\partial \mu}{\partial T}\right)_{P}=9.0
$$

These values give the temperature dependences of seismic velocities $(\partial \ln \alpha / \partial T)_{P}=-2.4 \times$ $10^{-5} \mathrm{~K}^{-1},(\partial \ln \beta / \partial T)_{P}=-4.8 \times 10^{-5} \mathrm{~K}^{-1}$. These temperature sensitivities are insufficient to explain reported lower mantle velocity anomalies without implausible temperature variations. Although temperature variations in the lower mantle must occur, tomographic observations do not provide direct evident for them, but are probably dominated by compositional variations. However, some correlation between composition and temperature may be expected, so that tomography may give still an indication of the pattern of the core-to-mantle heat flux.
\end{abstract}

\section{Introduction}

Certain properties of the geomagnetic field, such as the frequency of reversals and perhaps also the average dipole strength, vary on a time scale of $10^{8}$ years (CouRTILlot and BESSE, 1987; PRÉvot et al., 1990). Since this time scale does not arise naturally from core properties, but is characteristic of mantle convection we may seek an explanation in terms of mantle control of core processes. One possibility is a time-varying pattern of temperature gradients in the $\mathrm{D}^{\prime \prime}$ layer, at the base of the mantle, controlled by the flux of hot, softened material into mantle plumes (STACEY and LOPER, 1983). STACEY (1991, 1992, p. 321) suggested that this flow would cause rafts of dense material (crypto-continents) to move about on the core-mantle boundary (CMB), changing the areas of reduced boundary heat flux. The details of this mechanism are conjectural, but lateral temperature variations near the base of the mantle appear inevitable. The core-mantle boundary itself is maintained isothermal within about $10^{-5} \mathrm{~K}$ by the relatively rapid core motions (STACEY, 1991), so any slight variations in the lower mantle determine the variability of the core-to-mantle heat flux.

The $\mathrm{D}^{\prime \prime}$ layer itself is heterogeneous, with seismic velocity variations that must be attributed to compositional differences (crypto-continents?), masking any temperature effects. But we may suppose that the temperature variations extend further into the lower mantle and that evidence of them may be sought in seismic tomography. Central to this discussion are the temperature dependences of incompressibility, $K_{S}$, and rigidity, $\mu$, under lower mantle conditions, which are 
the subject of several recent discussions (AGNON and BUKOWINSKI, 1990; MAO et al., 1991; ISAAK et al., 1992; ANDERSON and ISAAK, 1993). They are represented by dimensionless parameters

$$
\begin{aligned}
\delta_{S} & =\frac{1}{\alpha_{V} K_{S}}\left(\frac{\partial K_{S}}{\partial T}\right)_{P}, \\
\epsilon & =\frac{1}{\alpha_{V} \mu}\left(\frac{\partial \mu}{\partial T}\right)_{P} .
\end{aligned}
$$

$\delta_{S}$ is known as the adiabatic Anderson-Grüneisen parameter and is accessible to thermodynamic analysis, as in the following section. There is no comparable constraint on $\epsilon$. The thermal interpretation of tomographic observations gives an $\mathrm{S}$-wave velocity, $\beta$, twice as sensitive to temperature, $T$, as the P-wave velocity, $\alpha$

$$
R=(\partial \ln \beta / \partial \ln \alpha)_{P} \approx 2 .
$$

However this cannot be taken to confirm that lower mantle velocity anomalies are thermal in origin. Moreover the inferred value of $(\partial \mu / \partial T)_{P}$ (at constant pressure, $P$ ) is so sensitive to the numerical value in Eq. (3) that almost any value of $(\partial \mu / \partial T)_{P}$ would be consistent with the observations.

A best-guessed ratio, $\left(\partial \ln \mu / \partial \ln K_{S}\right)_{P}=3.5$ to 4 , is insufficient to give lower mantle velocity variations of the observed magnitude without unreasonably strong large scale temperature variations. As STACEY (1992, p. 271) pointed out, an obvious possible explanation is that lower mantle velocity anomalies are dominated by compositional effects. In that case Eq. (3) says something about the compositional variations.

2. Temperature Dependence of Incompressibility: The Anderson-Grüneisen Parameters

We need a numerical value of $\left(\partial K_{S} / \partial T\right)_{P}$, which can be written in terms of accessible quantities by first writing

$$
\begin{aligned}
\left(\frac{\partial K_{S}}{\partial T}\right)_{P} & =\left(\frac{\partial K_{S}}{\partial T}\right)_{V}+\left(\frac{\partial K_{S}}{\partial V}\right)_{T}\left(\frac{\partial V}{\partial T}\right)_{P} \\
& =\left(\frac{\partial K_{S}}{\partial T}\right)_{V}+\left(\frac{\partial K_{S}}{\partial P}\right)_{T}\left(\frac{\partial P}{\partial V}\right)_{T}\left(\frac{\partial V}{\partial T}\right)_{P} \\
& =\left(\frac{\partial K_{S}}{\partial T}\right)_{V}-\alpha_{V} K_{T}\left(\frac{\partial K_{S}}{\partial P}\right)_{T}
\end{aligned}
$$

where $K_{T}$ is the isothermal incompressibility, $\alpha_{V}$ is volume expansion coefficient, with subscript $V$ introduced to avoid confusion with the use of $\alpha$ to denote $\mathrm{P}$-wave speed, and $T, V, P$ are temperature, volume and pressure. The first term on the right hand side of Eq. (4) can be written as a sum of four terms by differentiating the standard relationship

$$
K_{S}=K_{T}\left(1+\gamma \alpha_{V} T\right)
$$

where $\gamma=\alpha_{V} K_{T} / \rho C_{V}=\alpha_{V} K_{S} / \rho C_{P}$ is the thermodynamic Grüneisen parameter, $\rho$ is density and $C_{V}, C_{P}$ are the principal specific heats. Differentiation gives

$$
\begin{aligned}
\left(\frac{\partial K_{S}}{\partial T}\right)_{V} & =\frac{\partial}{\partial T_{V}}\left(K_{T}+\gamma \alpha_{V} K_{T} T\right) \\
& =\left(\frac{\partial K_{T}}{\partial T}\right)_{V}+\gamma T\left(\frac{\partial\left(\alpha_{V} K_{T}\right)}{\partial T}\right)_{V}+\alpha_{V} K_{T} T\left(\frac{\partial \gamma}{\partial T}\right)_{V}+\gamma \alpha_{V} K_{T}
\end{aligned}
$$


The three differentials on the right hand side of Eq. (6) are examples of second differential thermodynamic quantities, for which there is no convenient listing of identities similar to that for the first differentials (STACEY, 1977, 1992, p. 444). However, convenient expressions for these three are given by BRENNAN and STACEY (1979). With their equation numbers these are

$$
\text { B.\&S. Eq. (9): }\left(\frac{\partial K_{T}}{\partial T}\right)_{V}=\gamma \rho C_{V}\left[1-\left(\frac{\partial \ln C_{V}}{\partial \ln V}\right)_{T}-\left(\frac{\partial \ln \gamma}{\partial \ln V}\right)_{T}\right]
$$

where $\gamma \rho C_{V}=\alpha_{V} K_{T}$.

$$
\begin{gathered}
\text { B.\&S. Eq. (4): }\left(\frac{\partial\left(\alpha_{V} K_{T}\right)}{\partial T}\right)_{V}=\frac{\rho V}{T}\left(\frac{\partial C_{V}}{\partial V}\right)_{T} . \\
\text { B.\&S. Eq. (10): } T\left(\frac{\partial \gamma}{\partial T}\right)_{V}=\left(\frac{\partial \ln C_{V}}{\partial \ln V}\right)_{S} .
\end{gathered}
$$

Substituting these expressions in Eq. (6)

$$
\left(\frac{\partial K_{S}}{\partial T}\right)_{V}=\alpha_{V} K_{T}\left[1-\left(\frac{\partial \ln \gamma}{\partial \ln V}\right)_{T}+\left(\frac{\partial \ln C_{V}}{\partial \ln V}\right)_{S}+\gamma\right]
$$

and so

$$
\left(\frac{\partial K_{S}}{\partial T}\right)_{P}=\alpha_{V} K_{T}\left[1-\left(\frac{\partial \ln \gamma}{\partial \ln V}\right)_{T}+\left(\frac{\partial \ln C_{V}}{\partial \ln V}\right)_{S}+\gamma-\left(\frac{\partial K_{S}}{\partial P}\right)_{T}\right]
$$

Now

$$
\left(\frac{\partial K_{S}}{\partial P}\right)_{S}=\left(\frac{\partial K_{S}}{\partial P}\right)_{T}+\left(\frac{\partial K_{S}}{\partial T}\right)_{P}\left(\frac{\partial T}{\partial P}\right)_{S}=\left(\frac{\partial K_{S}}{\partial P}\right)_{T}+\left(\frac{\partial K_{S}}{\partial T}\right)_{P} \frac{\gamma T}{K_{S}}
$$

Using this to substitute for $\left(\partial K_{S} / \partial P\right)_{T}$ in Eq. (11), making $\left(\partial K_{S} / \partial T\right)_{P}$ the subject of the equation and then simplifying by Eq. (5), we obtain the final result

$$
\begin{aligned}
\delta_{S} & =\left(\frac{\partial \ln K_{S}}{\partial \ln \rho}\right)_{P}=-\frac{1}{\alpha_{V} K_{S}}\left(\frac{\partial K_{S}}{\partial T}\right)_{P} \\
& =\left(\frac{\partial K_{S}}{\partial P}\right)_{S}-\gamma-1+\left(\frac{\partial \ln \gamma}{\partial \ln V}\right)_{T}-\left(\frac{\partial \ln C_{V}}{\partial \ln V}\right)_{S}
\end{aligned}
$$

This is the adiabatic Anderson-Grüneisen parameter, which expresses $\left(\partial K_{S} / \partial T\right)_{P}$ in a conveniently dimensionless form.

More often quoted than $\delta_{S}$ is the corresponding isothermal Anderson-Grüneisen parameter, $\delta_{T}$, for which a similar expression can be obtained in terms of the same accessible quantities. The relationship between them is

$$
\begin{aligned}
\delta_{T}= & \delta_{S}(1+\gamma \alpha T)+\gamma+\left(\frac{\partial \ln C_{V}}{\partial \ln V}\right)_{S} \\
& +\left(\frac{\partial \ln C_{V}}{\partial \ln V}\right)_{T}(1+\gamma \alpha T)+\gamma \alpha T\left[2\left(\frac{\partial \ln \gamma}{\partial \ln V}\right)_{T}-1\right]
\end{aligned}
$$

It is clear that $\delta_{T}$ is substantially larger than $\delta_{S}$ and it is important not to use a numerical estimate of $\delta_{T}$ in assessing the temperature dependences of seismic velocities. 
In estimating a typical value of $\delta_{S}$ for the lower mantle from Eq. (13), STACEY $(1992$, p. 269) took $\left(\partial K_{S} / \partial P\right)_{S}=3.34, \gamma=0.96,(\partial \ln \gamma / \partial \ln V)=1$ and $\left(\partial \ln C_{V} / \partial \ln V\right)_{S}=0$, giving $\delta_{S}$ $=2.38$, admitting an uncertainty of \pm 0.5 , which is a generous estimate. The corresponding isothermal parameter is $\delta_{T}=3.44$. These values are consistent with the values obtained by ISAAK et al. (1992) and ANDERSON and ISAAK (1993) for MgO. They are not compatible with the much higher value of $\delta_{T}$ obtained for perovskite up to $30 \mathrm{GPa}$ by MAO et al. (1991). It is suggested that the value of $\delta_{S}$ by AGNON and BUKOwINSKI (1990) is slightly too low.

\section{Temperature Dependences of Seismic Velocities}

To estimate the effect of temperature on the seismic velocities $\alpha$ and $\beta$ we need, in addition to $\delta_{S}$, a numerical value for $\epsilon$ (Eq. (2)). Then by differentiating the expressions for velocity

$$
\begin{gathered}
\alpha=\sqrt{\left(K+\frac{4}{3} \mu\right) / \rho}, \\
\beta=\sqrt{\mu / \rho},
\end{gathered}
$$

we obtain

$$
\begin{gathered}
\left(\frac{\partial \ln \alpha}{\partial T}\right)_{P}=-\frac{\alpha_{V}}{2}\left[\frac{K_{S} \delta_{S}+\frac{4}{3} \mu \epsilon}{K_{S}+\frac{4}{3} \mu}-1\right], \\
\left(\frac{\partial \ln \beta}{\partial T}\right)_{P}=-\frac{\alpha_{V}}{2}(\epsilon-1)
\end{gathered}
$$

and therefore

$$
R=\left(\frac{\partial \ln \beta}{\partial \ln \alpha}\right)_{P}=\frac{\epsilon-1}{\left(K_{S} \delta_{S}+\frac{4}{3} \mu \epsilon\right) /\left(K_{S}+\frac{4}{3} \mu\right)-1} .
$$

Tomographic observations give $R \approx 2$ (ANDERson, 1987 ) so that with the value of $\delta_{S}$ obtained above and taking $\mu / K_{S}$ at a representative depth, $\epsilon \approx 9.0$. Although this appears reasonable it is far from being a secure estimate because $\epsilon$ appears in both the numerator and denominator of Eq. (19) and so, for large values, $R$ becomes insensitive to it. As $\epsilon \rightarrow \infty$, so $R \rightarrow\left[\left(K_{S}+\right.\right.$ $(4 / 3) \mu) /(4 / 3) \mu]=2.53$, which is not far removed from the favoured value. Also it must be noted that this estimate assumes the thermal interpretation of tomography which it is the purpose of this paper to question.

AGNON and BuKOWINSKI (1990) appealed to the identity

$$
\epsilon=\frac{K_{S}}{\mu}\left(\frac{\partial \mu}{\partial P}\right)_{S}-\frac{\left(1+\gamma \alpha_{V} T\right)}{\alpha_{V}}\left(\frac{\partial \ln \mu}{\partial T}\right)_{V}
$$

because the first term on the right hand side can be obtained directly from an earth model, such as PREM, if the lower mantle is assumed to be adiabatic. The numerical value, at the nominal depth of $1771 \mathrm{~km}$, as used here, is 2.36. This is certainly much smaller than the second term, which expresses the intrinsic temperature dependence of $\mu$. As YEGANEH-HAERI et al. (1989) noted, silicate perovskite, which is presumed to be the dominant lower mantle mineral, has an anomalously strong temperature dependence of $\mu$, which they tentatively attributed to a ferroelastic transition. In this circumstance $\epsilon$ cannot be effectively estimated from Eq. (20).

In the absence of better evidence, the value $\epsilon=9.0$, obtained above with the assumption that $R=2.0$ and that the lower mantle velocity anomalies are of thermal origin, may be used with Eqs. (17) and (18) and $\alpha_{V}=12 \times 10^{-6} \mathrm{~K}^{-1}$ to give

$$
\left(\frac{\partial \ln \alpha}{\partial T}\right)_{P}=-2.4 \times 10^{-5} \mathrm{~K}^{-1}
$$


Table 1. Values of $\epsilon$ (Eq. (2)) for different values of $\delta_{S}$ (Eq. (13)) and $R$ (Eq. (19)). The corresponding temperature increments for a $1 \%$ change in $\mathrm{P}$-wave velocity are given in parentheses.

\begin{tabular}{cccc}
\hline & \multicolumn{3}{c}{$\delta_{S}$} \\
\cline { 2 - 4 }$R$ & 1.9 & 2.38 & 2.9 \\
\hline 1.5 & 3.0 & 4.1 & 5.2 \\
& $(1247 \mathrm{~K})$ & $(813 \mathrm{~K})$ & $(591 \mathrm{~K})$ \\
2.0 & 6.2 & 9.0 & 11.9 \\
& $(643 \mathrm{~K})$ & $(419 \mathrm{~K})$ & $(304 \mathrm{~K})$ \\
2.5 & 110 & 168 & 231 \\
& $(38 \mathrm{~K})$ & $(25 \mathrm{~K})$ & $(18 \mathrm{~K})$ \\
\hline \multicolumn{4}{c}{} \\
$\left(\frac{\partial \ln \beta}{\partial T}\right)_{P}=-4.8 \times 10^{-5} \mathrm{~K}^{-1}$.
\end{tabular}

Thus a $1 \% \mathrm{P}$-wave velocity anomaly would be caused by a temperature increment of about 400 $\mathrm{K}$. The variations in this estimate obtained by choosing different values of $\delta_{S}$ and $R$ are indicated in Table 1.

\section{The Thermal Interpretation of Lower Mantle Velocity Anomalies}

Travel-time tomography of the lower mantle can resolve only large scale features. Taking the representation in spherical harmonics to degree 6 by DzIEwONSKI (1984) as representative of what is possible, the smallest identified features (half-wavelengths) correspond to about $2400 \mathrm{~km}$ in the middle of the lower mantle depth range. Thus, whatever the finer details, the implication is that there are velocity variations of order $0.5 \%$ averaged over at least this scale. If these variations are attributed to temperature then temperature variations $\Delta T= \pm 200 \mathrm{~K}$ are required and the corresponding density variations are $\Delta \rho= \pm 12.6 \mathrm{~kg} \mathrm{~m}^{-3}$. The estimated density contrast is not dependent on the assumed value of $\alpha_{V}$ because, as seen in Eq. (17), $\Delta \alpha$ depends on the product $\alpha_{V} \Delta T$ and so does $\Delta \rho / \rho$. The inferred density anomalies depend only on the square-bracketed term in Eq. (17), for which the central entry in Table 1 has been used.

There are several ways of examining the consequences of this result and all of them lead to the conclusion that an alternative to the simple thermal explanation is needed. One approach is to consider the effect of density anomalies on the geoid. Recognizing that the vertical resolution in tomographic results is greater than the horizontal resolution, we can consider the effect of a disc of radius $r$ at depth $z$ having a thickness $\Delta z \ll z$ and a density contrast $\Delta \rho$. It causes a deflection of the geoid given by

$$
\Delta h=2 \pi G \Delta \rho \Delta z\left[\left(z^{2}+r^{2}\right)^{1 / 2}-z\right] / g
$$

where $G$ is the gravitational constant and $g$ is ambient gravity. With $\Delta \rho=12.6 \mathrm{~kg} \mathrm{~m}^{-3}, z=1771$ $\mathrm{km}$ and $r>1200 \mathrm{~km}$ as considered above

$$
\Delta h>20(\Delta z / 100 \mathrm{~km}) \text { metres. }
$$

Since the geoid undulations attributable to the lower mantle are of order $50 \mathrm{~m}$ in amplitude, we are restricted to $\Delta z<250 \mathrm{~km}$. Since this is much smaller than the vertical extent of observed velocity anomalies the simple thermal explanation is inadequate

We may also consider the implication for mantle viscosity, $\eta$, of a large buoyant mass, rising at speed $v$, and equate the viscous drag to the buoyancy force

$$
6 \pi \eta r v=\pi r^{2} \Delta z \Delta \rho \cdot g .
$$


If $\eta<10^{22} \mathrm{~Pa}$ s then, with the dimensions considered above, $v>2.5 \times 10^{-9} \mathrm{~m} \mathrm{~s}^{-1}$ ( $8 \mathrm{~cm} /$ year), which is a quite implausible rate. Taking the heat content of moving material to be

$$
Q / V=\rho C_{P} \Delta T=1.3 \times 10^{9} \mathrm{~J} \mathrm{~m}^{-3}
$$

the consequent heat flux would be

$$
(Q / V) v>3.3 \mathrm{~W} \mathrm{~m}^{-2}
$$

some hundred times any plausible value.

\section{Conclusions}

Temperature variations must occur in the lower mantle and all that has been demonstrated here is that they are not uniquely related to tomographic velocity variations. It seems unlikely that the difficulty can be resolved by a sufficient revision of the material properties and there are therefore two options: to revise the velocity anomalies downwards or to attribute them to compositional variations. However, thermal properties of minerals are quite variable, so it is reasonable to expect some thermal variation at the base of the mantle to accompany compositional variations. Even a very slight effect would be sufficient to influence the flow of heat at the top of the core. Thus, it is still reasonable that the mantle heterogeneities should exert a subtle control on the core and that tomography should indicate the heterogeneities, but they should not be interpreted directly in terms of temperatures.

\section{REFERENCES}

Agnon, A. and M. S. T. Bukowinski, $\delta_{S}$ at high pressure and $d \ln V_{S} / d \ln V_{P}$ in the lower mantle, Geophys. Res. Lett., 17, 1149-1152, 1990.

Anderson, D. L., A seismic equation of state II. Shear properties and thermodynamics of the lower mantle, Phys. Earth Planet. Inter., 45, 307-323, 1987.

Anderson, O. L. and D. G. ISAAK, The dependence of the Anderson-Grüneisen parameter, $\delta_{T}$, upon compression at extreme conditions, J. Phys. Chem. Solids, 1993 (in press).

Brennan, B. J. and F. D. StACEY, A thermodynamically based equation of state for the lower mantle, J. Geophys. Res., 84, 5535-5539, 1979.

Courtillot, V. and J. Besse, Magnetic field reversals, polar wander and core-mantle coupling, Science, 237, 1140-1147, 1987.

DZIEWONSKI, A. M., Mapping the lower mantle: determination of lateral heterogeneity in $\mathrm{P}$ velocity up to degree and order 6, J. Geophys. Res., 89, 5929-5952, 1984.

ISAAK, D. G., O. L. ANDERSON, and R. E. COHEN, The relationship between shear and compressional velocities at high pressures: reconciliation of seismic tomography and mineral physics, Geophys. Res. Lett., 19, 741-744, 1992.

Mao, H. K., R. J. Hemley, Y. Fei, J. F. Shu, L. C. Chen, A. P. Jephcoat, Y. Wu, and W. A. Bassett, Effect of pressure, temperature and composition on lattice parameters and density of $(\mathrm{Fe}, \mathrm{Mg}) \mathrm{SiO}_{3}$-perovskites to $30 \mathrm{GPa}, J$. Geophys. Res., 96, 8069-8079, 1991.

Prévot, M., M. E. M. Derder, M. MCWilliams, and J. Thommpson, Intensity of the Earth's magnetic field: evidence for a Mesozoic dipole low, Earth Planet Sci. Lett., 97, 129-139, 1990.

STACEY, F. D., Applications of thermodynamics to fundamental earth physics, Geophys. Surv., 3, 175-204, 1977.

STACEY, F. D., Effects on the core of structure within D", Geophys. Astrophys. Fluid Dyn., 60, 157-163, 1991.

StACEY, F. D., Physics of the Earth, 3rd ed., 513 pp., Brookfield Press, Brisbane, 1992.

STACEY, F. D. and D. E. Loper, The thermal boundary layer interpretation of $\mathrm{D}^{\prime \prime}$ and its role as a plume source, Phys. Earth Planet. Inter., 33, 45-55, 1983.

YegANEh-HAeri, A., D. J. WeidNer, and E. ITo, Elasticity of $\mathrm{MgSiO}_{3}$ in the perovskite structure, Science, 243, 787-789, 1989. 